\title{
MicroRNA-296-5p downregulated AKT2 to inhibit hepatocellular carcinoma cell proliferation, migration and invasion
}

\author{
XIAOJUN MA, BAOXIANG ZHUANG and WENTAO LI \\ Morphological Laboratory, Clinical Medical College, Weifang Medical University, Weifang, Shandong 261053, P.R. China
}

Received April 18, 2016; Accepted March 17, 2017

DOI: $10.3892 / \mathrm{mmr} .2017 .6701$

\begin{abstract}
Hepatocellular carcinoma (HCC) is the fifth most common malignancy in men, and the seventh in women worldwide. Despite development in the therapy of $\mathrm{HCC}$, the prognosis of HCC patients remains poor. Therefore, it is of great significance to explore the molecular mechanism underlying HCC progression, and investigate novel therapeutic strategies for the treatments of HCC. MicroRNAs (miRs) are known to be involved in the pathogenesis of HCC. The present study aimed to investigate the expression patterns and potential roles of miR-296-5p in HCC. Results revealed that miR-296-5p was frequently downregulated in HCC tissue samples and cell lines. Additionally, reduced miR-296-5p expression levels were correlated with tumor size, TNM stage and metastasis in HCC. Gain-of-function demonstrated that miR-296-5p inhibited HCC cell proliferation, migration and invasion in vitro. Furthermore, AKT2 was identified as a novel direct and functional target of miR-296-5p in HCC. These findings indicated that miR-296-5p/AKT2 axis serves important roles in $\mathrm{HCC}$ carcinogenesis and progression, and miR-296-5p/AKT2 based target therapy hampers HCC tumor growth and metastasis.
\end{abstract}

\section{Introduction}

Hepatocellular carcinoma (HCC), the predominant subtype primary liver cancer, is the fifth most common malignancy in men and the seventh in women worldwide (1). Every year, there are 750,000 new cases and 700,000 deaths due to HCC around the world (2). Research in epidemiology has indicated that many risk factors contribute to the HCC occurrence and development, including hepatitis $\mathrm{B}$ virus or hepatitis $\mathrm{C}$ virus infection, dietary aflatoxin $\mathrm{B} 1$ contamination, chronic alcohol abuse and tobacco consumption, lack of dietary antioxidants,

Correspondence to: Professor Baoxiang Zhuang, Morphological Laboratory, Clinical Medical College, Weifang Medical University, 7166 Baotong West Road, Weifang, Shandong 261053, P.R. China E-mail: zhuang_baoxiang@163.com

Key words: miroRNA-296-5p, hepatocellular carcinoma, AKT2, target therapy arsenic exposure, obesity and non-alcoholic fatty liver disease (3). Other risks for HCC include geographic region, socio-economic status, gender, ethnicity and environmental exposures (4). Despite development in the therapy of HCC, the prognosis of HCC patients remains unsatisfactory (5). This is primarily due to the fact that only a small number of patients can receive surgery due to the limitations such as the stage of the HCC, the number and the size of the nodules, and the liver function (6). Additionally, the high rate of recurrence and metastasis after surgery also results in poor prognosis (7). Therefore, it is of great significance to explore the molecular mechanism underlying HCC progression, and investigate novel therapeutic strategies for the treatments of HCC.

MicroRNAs (miRs) are a large family of single strand, non-coding, and small RNAs consisting of 19-25 nucleotides (8). As a new type of gene expression regulators, miRNAs may regulate gene expression through base pairing with the 3' untranslated regions (3'UTRs) of their target genes and thus participate in a variety of biological and pathological processes, including cell proliferation, cycle, apoptosis, differentiation, metabolism, invasion and metastasis (9-11). Since the initial observation, $>1,000$ miRNAs have been validated, and are estimated to modulate the expression of $>60 \%$ of protein-coding genes (12). Accumulated studies have demonstrated that a larger number of miRNAs were dysregulated in human cancers with differential regulation ('up' or 'down') observed in neoplastic compared with normal cells (13). For example, miR-200b (14) and miR-379-5p (15) were downregulated, whereas miR-155 (16) and miR-130b (17) were upregulated in HCC. The involvement of miRNAs in cancer carcinogenesis and progression is well identified, as miRNAs can function as oncogenes or tumor suppressor genes depending on the roles of their target genes (18). Therefore, further exploration of the abnormal expressed miRNAs and their direct target genes would provide efficacious therapeutic targets for patients with HCC.

In the present study, the authors focused on the expression and roles of miR-296-5p in HCC. Results demonstrated that miR-296-5p was significantly downregulated in HCC, and reduced miR-296-5p expression levels were correlated with tumor size, TNM stage and metastasis. miR-296-5p overexpression suppressed cell proliferation, migration and invasion of HCC cells. Moreover, AKT2 was validated as the direct downstream and functional target gene of miR-296-5p in 
HCC. miR-296-5p acted as a tumor suppressor in HCC via direct targeting AKT2.

\section{Materials and methods}

Ethics statement and HCC samples. The current study was approved by the ethics committee of Weifang Medical University (Weifang, China). Written informed consent approving the use of their tissue samples for research purposes was also obtained from all the HCC patients. A total of $79 \mathrm{HCC}$ tissues and adjacent non-tumor liver tissues were obtained from patients underwent surgical resection of primary HCC at the Associated Hospital of Weifang Medical University (Weifang, China). All the tissue samples were histopathologically confirmed and stored in liquid nitrogen until use.

Cell lines, cell culture and transfection. Human HCC cell lines (HepG2, BEL-7402, SNU-182, SMMC-7721, Huh7), the human normal liver cell line L02 and HEK293T cell line were obtained from American Type Culture Collection (ATCC; Manassas, VA, USA). All cell lines were cultured according to ATCC's recommendations.

miR-296-5p mimics, miRNA negative control (NC), and the small interfering (si)RNAs targeted for AKT2 (AKT2 siRNA) and NC (NC siRNA) were synthesized by Guangzhou RiboBio Co., Ltd. (Guangzhou, China). For transfection, cells were seeded in six-well plates at a density of $8 \times 10^{5}$ cells per well and cultured overnight. miRNA or siRNA was transfected into cells by using Lipofectamine 2000 (Invitrogen; Thermo Fisher Scientific, Inc., Waltham, MA, USA), according to manufacturer's instructions. At $48 \mathrm{~h}$ following transfection, the transfection efficiency of miRNA and siRNA was determined by reverse transcription-quantitative polymerase chain reaction (RT-qPCR).

$R N A$ isolation and RT-qPCR. Total RNA was isolated from the cell lines or tissue samples using TRIzol reagent (Invitrogen; Thermo Fisher Scientific, Inc.), following to the manufacturer's protocol. Total RNA was reversed transcription into cDNA by using M-MLV Reverse Transcription system (Promega Corporation, Madison, WI, USA). Expression levels of miR-296-5p were determined by SYBR premix Ex Taq ${ }^{\mathrm{TM}}$ kit (Takara Biotechnology Co., Ltd., Dalian, China) and internal control of U6 RNA. AKT2 mRNA expression was quantified using SYBR Green PCR Master Mix (Applied Biosystems; Thermo Fisher Scientific, Inc.) with GADPH as an internal control. All RT-qPCR was performed on an ABI PRISM ${ }^{\circledR}$ 7700 Sequence Detection System (Applied Biosystems; Thermo Fisher Scientific, Inc.). Relative expression of miRNA and mRNA was evaluated as folder changes using $2^{-\Delta \Delta C a}$ method (19).

Cell Counting Kit 8 (CCK8) assay. Cell proliferation was determined with CCK8 (Dojindo Molecular Technologies, Inc., Kumamoto, Japan) assay. In briefly, transfected cells were harvested at $24 \mathrm{~h}$ post-transfection, and seeded at a density of 3,000 cells/well in 96-well plates. Cells were incubated for different periods $(0,24,48,72$ and $96 \mathrm{~h})$ in a humidified atmosphere containing $5 \% \mathrm{CO}_{2}$ at $37^{\circ} \mathrm{C}$. Subsequently, $10 \mu \mathrm{l} \mathrm{CCK} 8$ solution was added in each well and incubated for additional
$2 \mathrm{~h}$. The absorbance was detected at $450 \mathrm{~nm}$ using a microplate reader (Bio-Rad Laboratories, Inc., Hercules, CA, USA). Each experiment was repeated at least three times.

Transwell migration and invasion assay. Transwell migration and invasion assays were performed by using 24-well Transwell chambers (EMD Millipore, Billerica, MA, USA) with an $8 \mathrm{~mm}$ polycarbonate membrane. For the Transwell invasion assay, the polycarbonate membranes were coated with Matrigel (BD Biosciences, Franklin Lakes, NJ, USA). Briefly, $5 \times 10^{4}$ transfected cells in $200 \mu \mathrm{l}$ fetal bovine serum (FBS; Gibco; Thermo Fisher Scientific, Inc.)-free culture medium were placed into the upper chamber, and the lower chamber was filled with $500 \mu \mathrm{l}$ culture medium containing $20 \%$ FBS. After incubation for $48 \mathrm{~h}$ in a humidified atmosphere containing $5 \% \mathrm{CO}_{2}$ at $37^{\circ} \mathrm{C}$, the cells on the upper chamber of the polycarbonate membranes were wiped off by cotton bud. The migrated and invaded cells were fixed in 4\% paraformaldehyde (Beyotime Institute of Biotechnology, Haimen, China), stained with $0.5 \%$ crystal violet (Beyotime Institute of Biotechnology), and washed three times with phosphate-buffered saline (PBS; Gibco; Thermo Fisher Scientific, Inc.). Finally, cells in five microscopic fields were counted and photographed with a light microscope (Olympus Corporation, Tokyo, Japan).

Western blot analysis. Cellular proteins were isolated by using radioimmunoprecipitation assay buffer (Beyotime Institute of Biotechnology). Proteins were resolved on 10\% SDS-PAGE and then transferred onto polyvinylidene fluoride (EMD Millipore) membranes. Subsequently, the membranes were blocked with 5\% skim milk in TBS containing $0.1 \%$ Tween 20 (TBST) at room temperature for $1 \mathrm{~h}$, and incubated with primary antibodies, mouse anti-human monoclonal AKT2 antibody (1:1,000 dilution; cat. no. sc-5270; Santa Cruz Biotechnology Inc., Dallas, TX, USA) and anti-human monoclonal GADPH antibody (1:1,000 dilution; cat. no. sc-365062; Santa Cruz Biotechnology, Inc.), at $4^{\circ} \mathrm{C}$ overnight. On the following day, the membranes were washed in TBST three times, and treated with corresponding goat anti-mouse horseradish peroxidase-conjugated secondary antibody $(1: 1,000$ dilution; cat. no. sc-2005; Santa Cruz Biotechnology, Inc.) diluted in TBST at room temperature for $1 \mathrm{~h}$. After washing in TBST, the signals were detected with enhanced chemiluminescence system Western Blotting Detection Reagents (GE Healthcare Life Sciences, Chalfont, UK) and photographed by FluorChem imaging system (version 4.1.0; Alpha Innotech, San Leandro, CA, USA).

Luciferase reporter assay. For the luciferase reporter assay, luciferase reporter vectors (pMIR-AKT2-3'UTR Wt 1, pMIR-AKT2-3'UTR Wt 2, pMIR-AKT2-3'UTR Mut 1 and pMIR-AKT2-3'UTR Mut 2) were synthesized by Shanghai GenePharma Co., Ltd. (Shanghai, China). HEK293T cells were seeded in 24-well plates at a density of $1.5 \times 10^{5}$ cells per well and cotransfected with pMIR-AKT2-3'UTR Wt (1 and 2) or pMIR-AKT2-3'UTR Mut (1 and 2) and miR-296-5p mimics or NC using Lipofectamine 2000. At 48 h post-transfection, cells were harvested and luciferase activities were measured by using Dual-Luciferase Reporter Assay System (Promega Corporation), following to the manufacturer's protocol. Firefly 
Table I. Correlations between miR-296-5p expression and clinicopathological characteristics in hepatocellular carcinoma.

\begin{tabular}{|c|c|c|c|c|}
\hline \multirow{2}{*}{$\begin{array}{l}\text { Clinical } \\
\text { features }\end{array}$} & \multirow{2}{*}{$\begin{array}{c}\text { Case } \\
\text { number }\end{array}$} & \multicolumn{2}{|c|}{$\begin{array}{l}\text { miR-296-5p } \\
\text { expression }\end{array}$} & \multirow[b]{2}{*}{ P-value } \\
\hline & & Low & High & \\
\hline Age & & & & 0.666 \\
\hline$<50$ years & 44 & 23 & 21 & \\
\hline$\geq 50$ years & 35 & 20 & 15 & \\
\hline Gender & & & & 0.849 \\
\hline Male & 54 & 29 & 25 & \\
\hline Female & 25 & 14 & 11 & \\
\hline Tumor size & & & & 0.005 \\
\hline$<5 \mathrm{~cm}$ & 39 & 15 & 24 & \\
\hline$\geq 5 \mathrm{~cm}$ & 40 & 28 & 12 & \\
\hline Tumor number & & & & 0.789 \\
\hline Single & 47 & 25 & 22 & \\
\hline Multiple & 32 & 18 & 14 & \\
\hline TNM Stage & & & & 0.013 \\
\hline I-II & 30 & 11 & 19 & \\
\hline III-IV & 49 & 32 & 17 & \\
\hline Metastasis & & & & 0.033 \\
\hline No & 59 & 28 & 31 & \\
\hline Yes & 20 & 15 & 5 & \\
\hline Differentiated & & & & 0.975 \\
\hline High & 55 & 30 & 25 & \\
\hline Low & 24 & 13 & 11 & \\
\hline
\end{tabular}

miR, microRNA.

luciferase activities were normalized to Renilla luciferase activities. Each sample was performed in triplicate.

Statistical analysis. Data were presented as mean \pm standard deviation, and compared using Student's t-test in SPSS 16.0 statistical software (SPSS, Inc., Chicago, IL, USA). P<0.05 was considered to indicate a statistically significant difference.

\section{Results}

miR-296-5p was downregulated in HCC tissues and cell lines. To explore the roles of miR-296-5p in HCC, its expression in HCC tissues and adjacent non-tumor liver tissues was measured by using RT-qPCR. The results demonstrated that miR-296-5p was obvious downregulated in HCC tissues compared with that in adjacent non-tumor liver tissues (Fig. 1A; $\mathrm{P}<0.05$ ).

To validate whether the abnormal expression of miR-296-5p was also the case in HCC cell lines, its expression in five HCC cell lines was then detected (HepG2, BEL-7402, SNU-182, SMMC-7721 and Huh7), as well as the human normal liver cell line L02. The results confirmed that miR-296-5p expression levels were reduced in each HCC cell lines than in L02 (Fig. 1B; P<0.05).
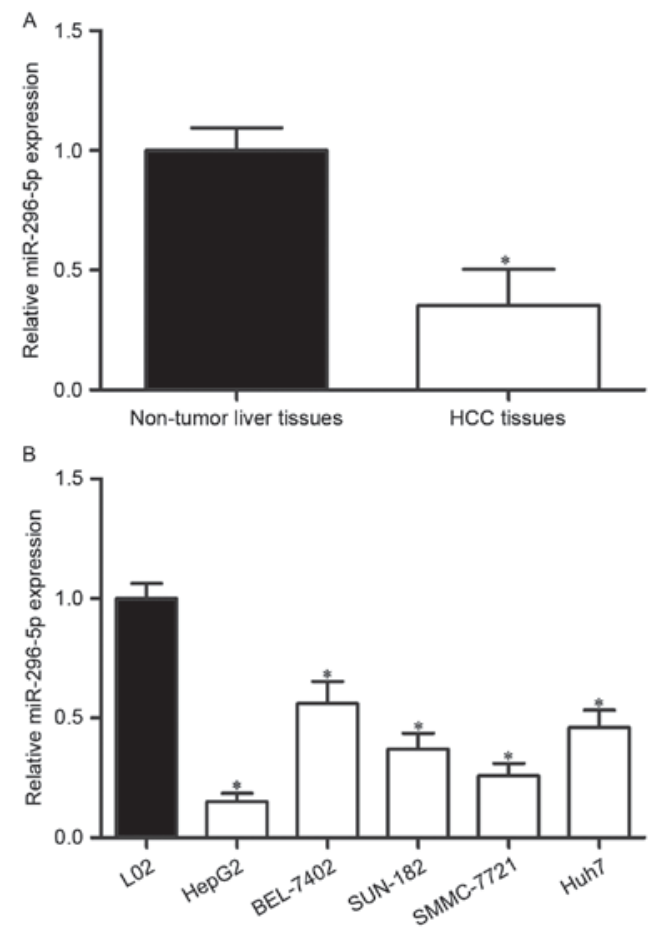

Figure 1. miR-296-5p expression levels in HCC. (A) Measurement of miR-296-5p levels in HCC tissues and adjacent non-tumor liver tissues by using RT-qPCR. (B) RT-qPCR analysis of miR-296-5p expression in five HCC cell lines, HepG2, BEL-7402, SNU-182, SMMC-7721 and Huh7, against the human normal liver cell line L02. ${ }^{*} \mathrm{P}<0.05$ vs. respective control. miR, microRNA; HCC, hepatocellular carcinoma; RT-qPCR, reverse transcription-quantitative polymerase chain reaction.

The relationship between miR-296-5p expression and clinicopathological characteristics in HCC. The clinicopathological characteristics of miR-296-5p expression in patients with HCC was investigated. As presented in Table I, miR-296-5p expression levels were significantly correlated with tumor size $(\mathrm{P}=0.005)$, TNM stage $(\mathrm{P}=0.013)$ and metastasis $(\mathrm{P}=0.033)$, but not with other clinicopathological characteristics, including age $(\mathrm{P}>0.05)$, gender $(\mathrm{P}>0.05)$, tumor number $(\mathrm{P}>0.05)$ and differentiation $(\mathrm{P}>0.05)$, in patients with $\mathrm{HCC}$.

miR-296-5p inhibited the proliferation, migration and invasion of HCC cells in vitro. To determine the functions of miR-296-5p on HCC progression, the authors transfected HepG2 and SMMC-7721 cells with miR-296-5p mimics or NC. Following transfection, RT-qPCR reported that miR-296-5p was remarkably increased in both HepG2 and SMMC-7721 cells transfected with miR-296-5p mimics (Fig. 2A; $\mathrm{P}<0.05$ ).

Considering that miR-296-5p expression was correlated with tumor size, the authors explored the effect of miR-296-5p on HCC proliferation using CCK8 assay. As demonstrated in Fig. 2B, restoration of miR-296-5p expression resulted in the inhibition of HepG2 and SMMC-7721 cells proliferation $(\mathrm{P}<0.05)$. Furthermore, given the association between miR-296-5p expression and HCC metastasis, Transwell migration and invasion assays were performed to evaluate the effects of miR-296-5p on HCC cells metastasis. It was observed that enforced miR-296-5p expression decreased the migration and invasion abilities in HepG2 and SMMC-7721 cells (Fig. 2C; 

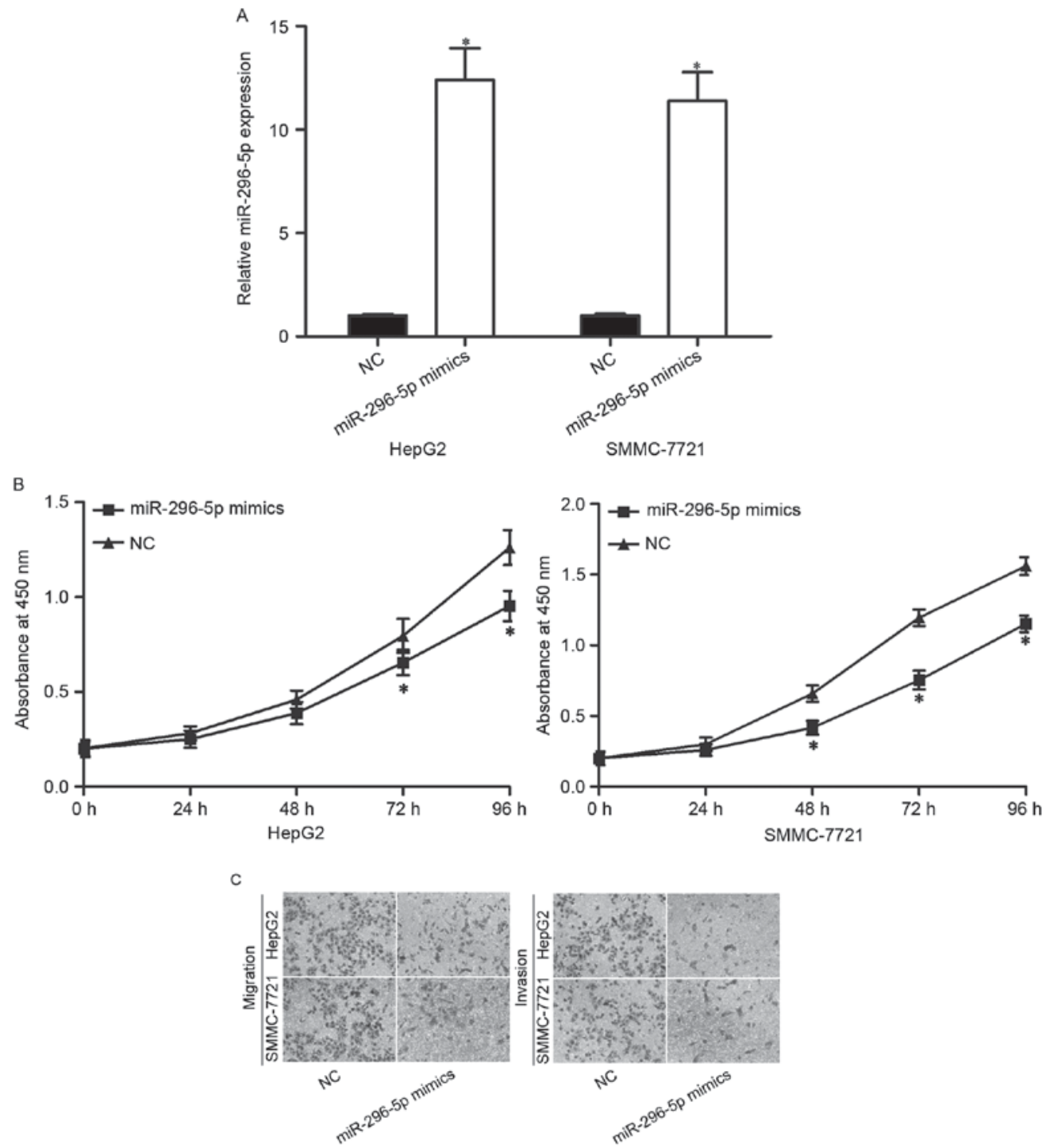

Figure 2. miR-296-5p overexpression inhibited HCC cells proliferation, migration and invasion. (A) Reverse transcription-quantitative polymerase chain reaction was performed to determine the expression of miR-296-5p in HepG2 and SMMC-7721 cells transfected with miR-296-5p mimics or NC. (B) The Cell Counting Kit-8 assay presented cell proliferation inhibition in miR-296-5p mimics compared with the NC from HepG2 and SMMC-7721 cells. (C) Transwell migration and invasion assay revealed cell migration and invasion inhibition in miR-296-5p mimics trasfected-HepG2 and SMMC-7721 cells. "P<0.05 vs. respective control. miR, microRNA; HCC, hepatocellular carcinoma; NC, negative control.

$\mathrm{P}<0.05)$. These results demonstrated that miR-296-5p acted as a tumor suppressor in HCC, through inhibiting cellular growth and metastasis in vitro.

AKT2 was a direct target gene of miR-296-5p. To further explore the molecular mechanism of miR-296-5p mediated suppressive roles in HCC, the authors analyzed the direct target genes of miR-296-5p. Firstly, TargetScan (http://www. targetscan.org) and miRanda (http://www.microrna.org) were used to predicate the potential target genes of miR-296-5p. The analyses suggested that 3'UTR of AKT2 contained the highly conserved putative miR-296-5p binding sites (Fig. 3A). Subsequently, RT-qPCR and western blot analysis were performed. The results indicated that upregulation of miR-296-5p suppressed AKT2 expression in HepG2 and SMMC-7721 cells at both mRNA (Fig. 3B; P<0.05) and protein (Fig. 3C; $\mathrm{P}<0.05$ ) levels. Finally, luciferase reporter assay was adopted to validate direct targeting of AKT2 by miR-296-5p.
As demonstrated in Fig. 3D and E, the luciferase activities were significantly reduced in HEK293T cells co-transfected with pMIR-AKT2-3'UTR Wt (1 and 2) and miR-296-5p mimics. However, miR-296-5p did not significantly alleviate the luciferase activities in HEK293T cells transfected with pMIR-AKT2-3'UTR Mut (1 and 2). Taken together, these results strongly demonstrated that AKT2 was a direct downstream target of miR-296-5p in HCC.

AKT2 was a downstream mediator of the biological roles of miR-296-5p in HCC. To investigate whether AKT2 mediated the biological roles of miR-296-5p in HCC, AKT2 siRNA was adopted to knockdown AKT2 expression in HepG2 and SMMC-7721 cells. Following transfection, RT-qPCR and western blot analysis revealed that AKT2 siRNA could reduced AKT2 mRNA (Fig. 4A; $\mathrm{P}<0.05$ ) and protein (Fig. 4B; $\mathrm{P}<0.05)$ expression levels in HepG2 and SMMC-7721 cells compared with NC siRNA groups. 
A

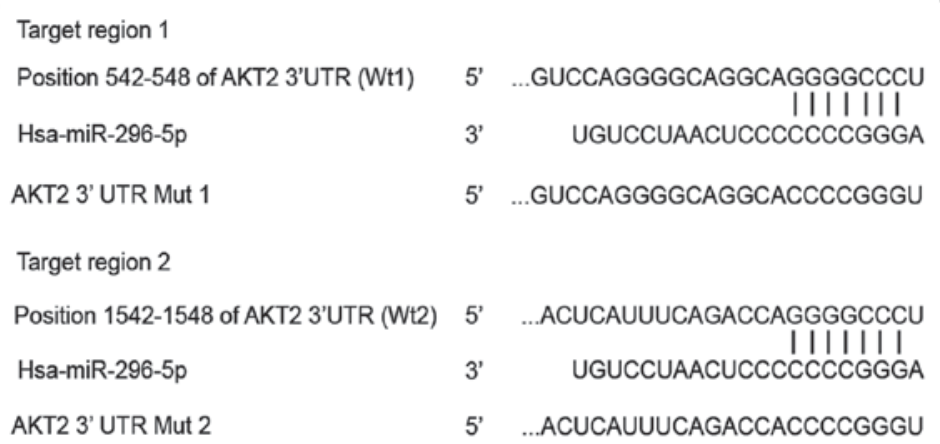

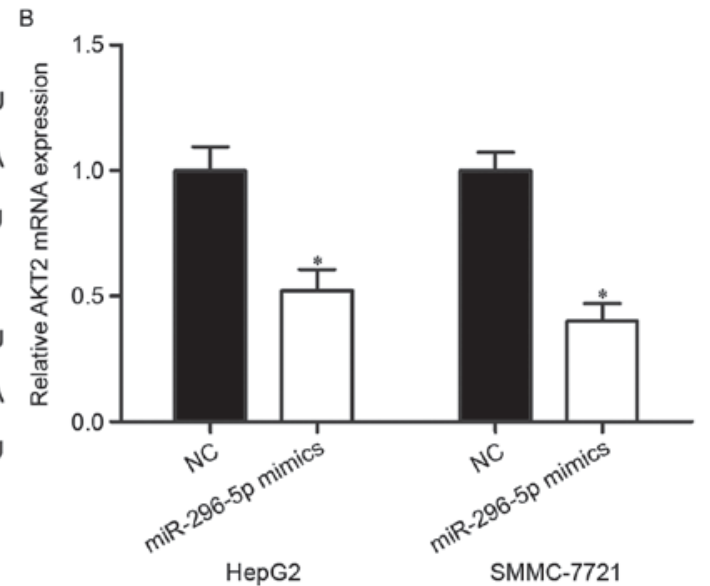

D

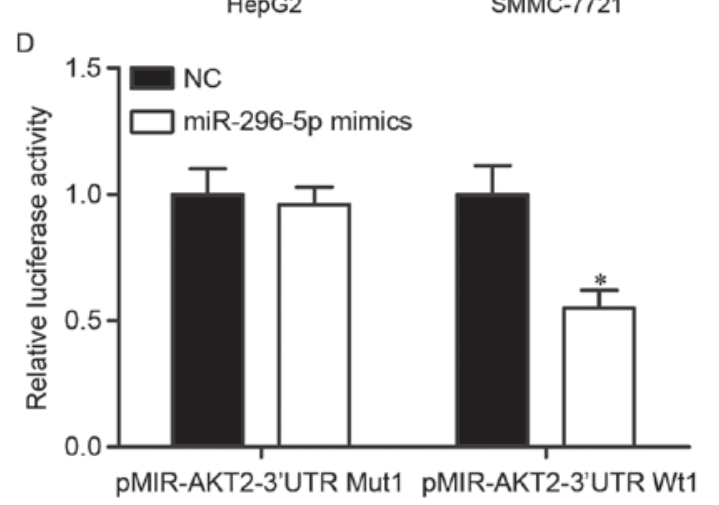

PMIR-AKT2-3'UTR Wt1
C

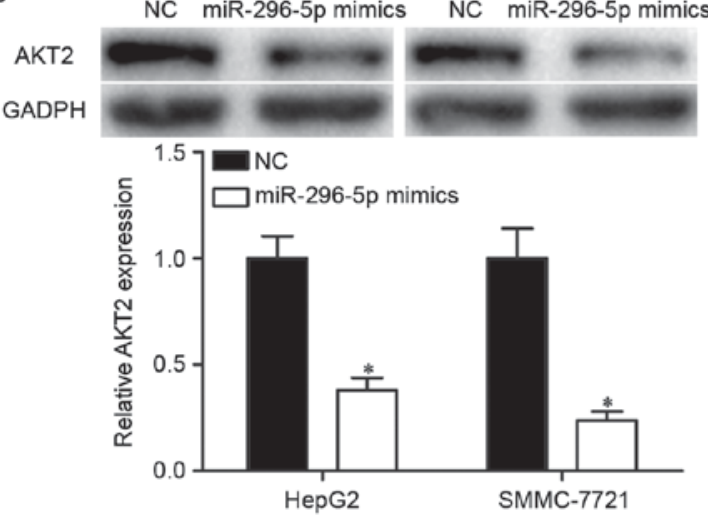

E

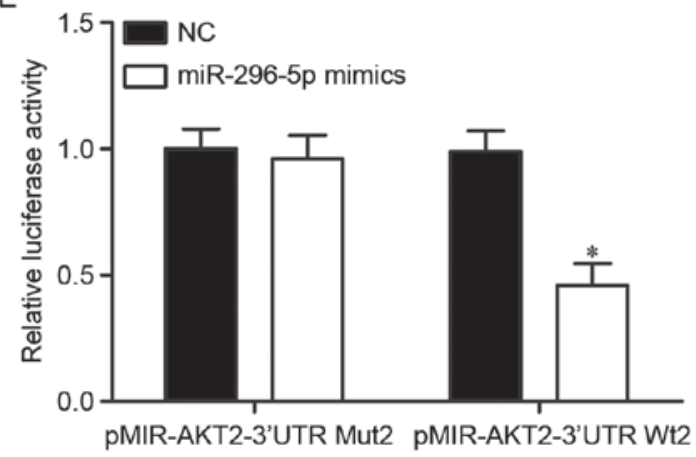

Figure 3. miR-296-5p directly targeted AKT2 in HCC. (A) The binding sites of miR-296-5p on 3' UTR of AKT2 was presented. The mutated binding sequence of AKT2 3'UTR was also shown. (B) After transfection with miR-296-5p mimics or NC, AKT2 mRNA expression levels in HepG2 and SMMC-7721 cells were measured by reverse transcription-quantitative polymerase chain reaction. (C) AKT2 protein expression was also detected in miR-296-5p overexpressed HepG2 and SMMC-7721 cells. (D and E) HEK293T cells were co-transfected with miR-296-5p mimics or NC, and pMIR-AKT2-3'UTR Wt (1 and 2) or pMIR-AKT2-3'UTR Mut (1 and 2). At $48 \mathrm{~h}$ following transfection, the relative luciferase activities were measured using the Dual-Luciferase Reporter Assay System. "P<0.05 vs. respective control. miR, microRNA; HCC, hepatocellular carcinoma; UTR, untranslated region; NC, negative control.

The CCK8 assay presented that the proliferation ability of the HepG2 and SMMC-7721 cells transfected with AKT2 siRNA was markedly lower than that of the NC siRNA groups (Fig. 4C; $\mathrm{P}<0.05$ ). Moreover, Transwell migration and invasion assays indicated that silencing of AKT2 led to a significant reduction of migration and invasion capacities in HepG2 and SMMC-7721 cells (Fig. 4D; P<0.05). These results were consistent with the findings that miR-296-5p overexpression can inhibit HCC cells proliferation, migration and invasion in vitro, which provided further evidences that AKT2 was a downstream mediator of the biological roles of miR-296-5p in HCC.

\section{Discussion}

Increasing studies demonstrated that the abnormal expression of miRNAs may be involved in HCC occurrence and development, and some of these miRNAs may act as tumor suppressors or oncogenes (20-22). Therefore, a better understanding of the specific molecular events involved by miRNAs correlated with carcinogenesis and progression of HCC is significant in exploring new therapeutic strategies to improve the prognosis of patients with this disease. In the present study, for the first time, the authors revealed the expression and roles of miR-296-5p in HCC. miR-296-5p was obvious 

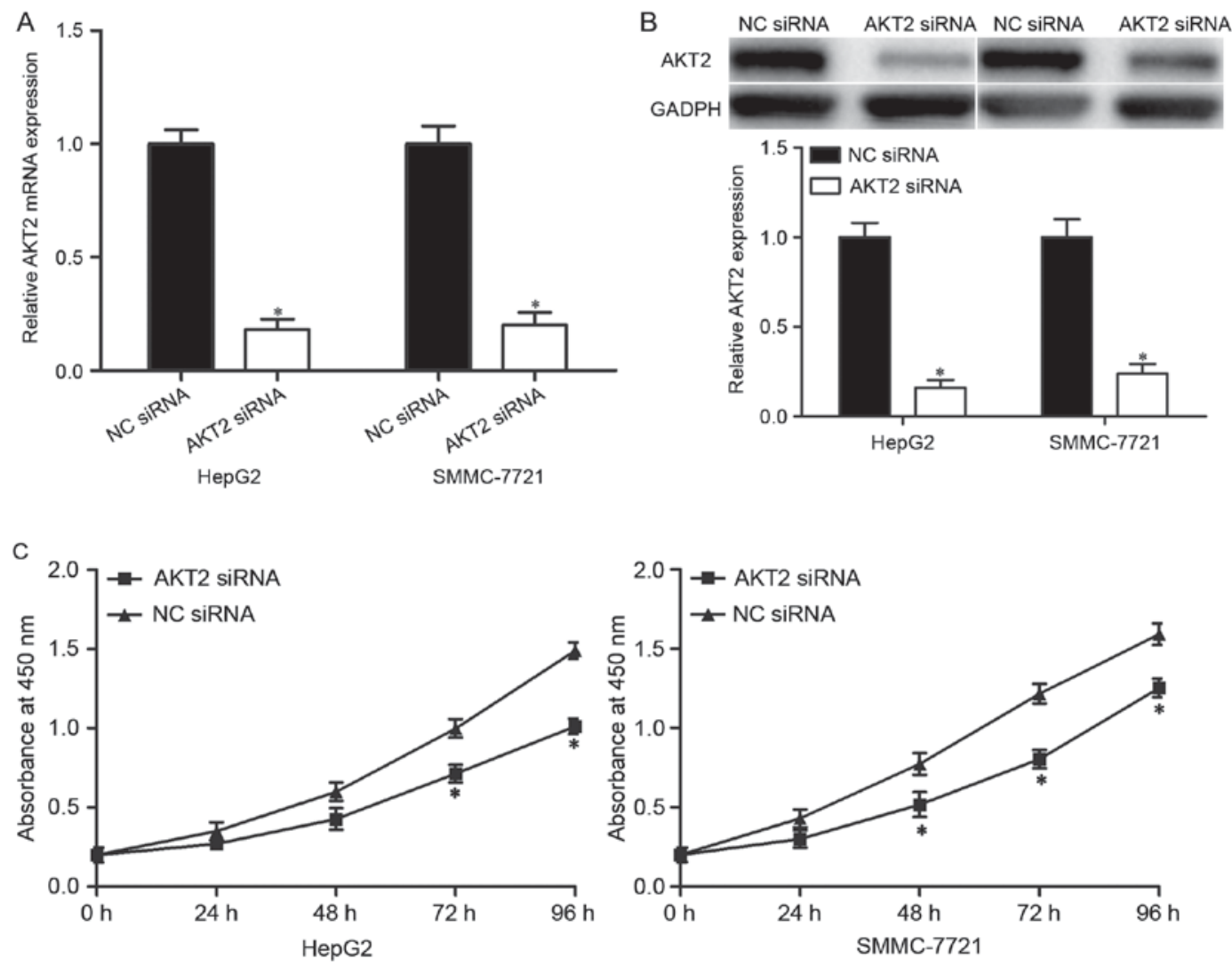

D
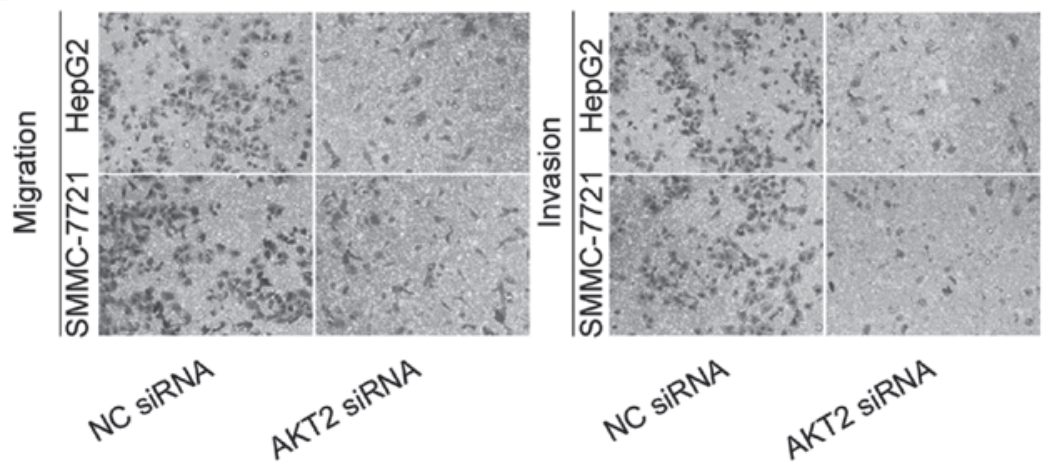

Figure 4. AKT2 siRNA inhibited HCC cells proliferation, migration and invasion. (A) AKT2 mRNA was examined by reverse transcription-quantitative polymerase chain reaction in HepG2 and SMMC-7721 cells treated with AKT2 siRNA or NC siRNA. (B) AKT2 protein levels were measured by western blotting in HepG2 and SMMC-7721 cells treated with AKT2 siRNA or NC siRNA. (C) CCK8 assay was performed on HepG2 and SMMC-7721 cells treated with AKT2 siRNA or NC siRNA. (D) Transwell migration and invasion assays were conducted in HepG2 and SMMC-7721 cells treated with AKT2 siRNA or NC siRNA. "P<0.05 vs. respective control. siRNA, small interfering RNA; HCC, hepatocellular carcinoma; NC, negative control.

downregulated in HCC tissues and cell lines compared with adjacent non-tumor liver tissues and human normal liver cell line, respectively. Reduced miR-296-5p levels were correlated with tumor size, TNM stage and metastasis in HCC. The gain-of-function demonstrated that miR-296-5p suppressed cell proliferation, migration and invasion of HCC. Mechanistically, AKT2 was identified as a direct target gene of miR-296-5p via bioinformatics analysis, RT-qPCR, western blotting and luciferase reporter assay. Furthermore, the knockdown of AKT2 may phenocopy the suppressive roles of miR-296-5p overexpression in HCC. These results indicated that miR-296-5p may be a novel therapeutic target for preventing HCC from rapidly growth and metastasis.
It has been reported that miR-296-5p was dysregulated in diverse cancer types. For example, in breast cancer, miR-296-5p was downregulated in tumor tissues, and low expression levels of miR-296-5p predicted shorter disease-free survival independently of classic clinicopathological parameters. In addition, reduced miR-296-5p was associated with an earlier spread of breast cancer in the overall series and with distant metastases in the subset (23). The downregulation of miR-296-5p was also identified in non-small cell lung cancer (24) and prostate cancer (25). However, miR-296-5p expression was upregulated in esophageal cancer tissues, and low expression of miR-296-5p was able to distinguish long-term survivors with node-positive disease from those 
dying within 20 months by predicting survival (26). Gastric cancer also expressed high levels of miR-296-5p (27). The present study indicated that miR-296-5p was downregulated in HCC, and correlated with pathological factors in HCC. Altogether, miR-296-5p expression has tissue specificity, and may be a prognostic marker for HCC.

The functions of miR-296-5p were mainly reported in human cancer research. In non-small cell lung caner, miR-296-5p targeted PLK1 to inhibit cell viability (24). In prostate cancer, miR-296-5p overexpression decreased cell growth and invasion capacity by negative regulation of HMGA1 (28). Lee et al (25) also reported that upregulation of miR-296-5p suppressed cell proliferation and anchorage-independent growth of prostate cancer cell lines through targeting Pin1. Savi et al (23) revealed that restoration of miR-296-5p into tumors of a breast cancer xenograft model significantly decreased tumor growth via directly targeting SCRIB. These findings verified that miR-296-5p may act as a tumor suppressor in tumorigenesis and tumor development. Oppositely, it was demonstrated to act as an oncogene $(26,27)$. In esophageal cancer, miR-296-5p underexpression repressed cell proliferation in vitro and in vivo via targeting cyclin D1 and p27. Furthermore, downregulation of miR-296-5p could confer sensitivity of both P-glycoprotein-related and P-glycoprotein-nonrelated drugs on esophageal cancer cells, and may promote ADR-induced apoptosis, accompanied by increased accumulation and decreased releasing amount of ADR (26). Li and his colleagues (27) reported that enforced miR-296-5p expression promoted cell proliferation in gastric cancer through downregulation of CDX1. These conflicting findings revealed that the roles of miR-296-5p have tissue specificity, and could be explained by the 'imperfect complementarity' of the interactions between miR-296-5p and their direct target genes.

miRs serve significant roles in carcinogenesis and progression of cancer by targeting key regulator. In addition, miRs can act as either oncogenes or tumor suppressors, primarily depending on the functions of their target genes in cancer. Therefore, in the present work, the mechanism by which miR-296-5p inhibited HCC cells proliferation, migration and invasion was explored. In the current study, AKT2 was identified as a novel direct downstream and functional target of miR-296-5p in HCC. AKT is a key element in PI3K/AKT pathway. The PI3K/AKT pathway is associated with aggressive phenotypes and poor outcomes in many human cancers (29). In addition, the PI3K/AKT pathway involves in a great deal of cellular processes such as cell proliferation, apoptosis, migration, invasion and metabolism $(30,31)$. A previous study verified that AKT2 was upregulated in HCC tissues, and expression levels of AKT2 were associated with histopathological differentiation, portal invasion and number of tumor nodules in HCC (32). Multivariate analysis also revealed that AKT2 was an independent prognostic marker for patients with HCC (32). A recent studies also indicated that AKT2 may be regulated by multiple miRs in HCC. For example, miR-302b targeted AKT2 to inhibit HCC cells invasion and metastasis (33). miR-137 suppressed HCC cells growth and metastasis through downregulation of AKT2 (34). Taken together, these data provided solid evidence to support that the miRs/AKT2 axis is a potential therapeutic target in HCC.
In conclusion, the current study indicated that miR-296-5p acted as a tumor suppressor in HCC, through inhibiting cell proliferation, migration and invasion.

The miR-296-5p/AKT2 axis serves important roles in HCC carcinogenesis and progression, and miR-296-5p/AKT2 based targeted therapy hampers HCC tumor growth and metastasis. Restoration of miR-296-5p may represent a new therapeutic strategy for patients with HCC. However, the molecular mechanism of low expression of miR-296-5p in HCC requires further research.

\section{References}

1. Ferlay J, Shin HR, Bray F, Forman D, Mathers C and Parkin DM: Estimates of worldwide burden of cancer in 2008: GLOBOCAN 2008. Int J Cancer 127: 2893-2917, 2010.

2. Borel F, Konstantinova P and Jansen PL: Diagnostic and therapeutic potential of miRNA signatures in patients with hepatocellular carcinoma. J Hepatol 56: 1371-1383, 2012.

3. Yu MC and Yuan JM: Environmental factors and risk for hepatocellular carcinoma. Gastroenterology 127 (5 Suppl 1): S72-S78, 2004.

4. El-Serag HB and Rudolph KL: Hepatocellular carcinoma: Epidemiology and molecular carcinogenesis Gastroenterology 132: 2557-2576, 2007.

5. Bi Q, Tang S, Xia L, Du R, Fan R, Gao L, Jin J, Liang S, Chen Z, $\mathrm{Xu} \mathrm{G}$, et al: Ectopic expression of MiR-125a inhibits the proliferation and metastasis of hepatocellular carcinoma by targeting MMP11 and VEGF. PLoS One 7: e40169, 2012.

6. Karaman B, Battal B, Sari S and Verim S: Hepatocellular carcinoma review: Current treatment and evidence-based medicine. World J Gastroenterol 20: 18059-18060, 2014.

7. Jonas S, Bechstein WO, Steinmüller T, Herrmann M, Radke C, Berg T, Settmacher U and Neuhaus P: Vascular invasion and histopathologic grading determine outcome after liver transplantation for hepatocellular carcinoma in cirrhosis. Hepatology 33: 1080-1086, 2001.

8. Lagos-Quintana M, Rauhut R, Lendeckel W and Tuschl T: Identification of novel genes coding for small expressed RNAs. Science 294: 853-858, 2001.

9. Kuhn AR, Schlauch K, Lao R, Halayko AJ, Gerthoffer WT and Singer CA: MicroRNA expression in human airway smooth muscle cells: Role of miR-25 in regulation of airway smooth muscle phenotype. Am J Respir Cell Mol Biol 42: 506-513, 2010.

10. Hwang HW and Mendell JT: MicroRNAs in cell proliferation, cell death, and tumorigenesis. Br J Cancer 96 (Suppl): R40-R44, 2007.

11. Kato M and Slack FJ: microRNAs: Small molecules with big roles - C. elegans to human cancer. Biol Cell 100: 71-81, 2008.

12. Esquela-Kerscher A and Slack FJ: Oncomirs-microRNAs with a role in cancer. Nat Rev Cancer 6: 259-269, 2006.

13. Calin GA and Croce CM: MicroRNA signatures in human cancers. Nat Rev Cancer 6: 857-866, 2006.

14. Li XY, Feng XZ, Tang JZ, Dong K, Wang JF, Meng CC, Wang J, Mo YW and Sun ZW: MicroRNA-200b inhibits the proliferation of hepatocellular carcinoma by targeting DNA methyltransferase 3a. Mol Med Rep 13, 3929-3935: 2016.

15. Chen JS, Li HS, Huang JQ, Dong SH, Huang ZJ, Yi W, Zhan GF, Feng JT, Sun JC and Huang XH: MicroRNA-379-5p inhibits tumor invasion and metastasis by targeting FAK/AKT signaling in hepatocellular carcinoma. Cancer Lett 375: 73-83, 2016.

16. Guan C, Yang F, He X, Li T, Yang Q, He H and Xu M: Clinical significance of microRNA-155 expression in hepatocellular carcinoma. Oncol Lett 11: 1574-1580, 2016.

17. Chang RM, Xu JF, Fang F, Yang H and Yang LY: MicroRNA-130b promotes proliferation and EMT-induced metastasis via PTEN/ p-AKT/HIF-1 $\alpha$ signaling. Tumour Biol 37: 10609-10619, 2016.

18. Bartel DP: MicroRNAs: Genomics, biogenesis, mechanism, and function. Cell 116: 281-297, 2004.

19. Livak KJ and Schmittgen TD: Analysis of relative gene expression data using real-time quantitative PCR and the 2(-Delta Delta C(T)) Method. Methods 25: 402-408, 2001.

20. Dong Y, Zou J, Su S, Huang H, Deng Y, Wang B and Li W: MicroRNA-218 and microRNA-520a inhibit cell proliferation by downregulating E2F2 in hepatocellular carcinoma. Mol Med Rep 12: 1016-1022, 2015. 
21. Chen X, Bo L, Zhao X and Chen Q: MicroRNA-133a inhibits cell proliferation, colony formation ability, migration and invasion by targeting matrix metallopeptidase 9 in hepatocellular carcinoma. Mol Med Rep 11: 3900-3907, 2015.

22. Li G, Yang F, Xu H, Yue Z, Fang X and Liu J: MicroRNA-708 is downregulated in hepatocellular carcinoma and suppresses tumor invasion and migration. Biomed Pharmacother 73: $154-159,2015$

23. Savi F, Forno I, Faversani A, Luciani A, Caldiera S, Gatti S, Foa P, Ricca D, Bulfamante G, Vaira V and Bosari S: miR-296/Scribble axis is deregulated in human breast cancer and miR-296 restoration reduces tumour growth in vivo. Clin Sci (Lond) 127: 233-242, 2014.

24. Xu C, Li S, Chen T, Hu H, Ding C, Xu Z, Chen J, Liu Z, Lei Z, Zhang HT, et al: miR-296-5p suppresses cell viability by directly targeting PLK1 in non-small cell lung cancer. Oncol Rep 35: 497-503, 2016.

25. Lee KH, Lin FC, Hsu TI, Lin JT, Guo JH, Tsai CH, Lee YC, Lee YC, Chen CL, Hsiao M and Lu PJ: MicroRNA-296-5p (miR-296-5p) functions as a tumor suppressor in prostate cancer by directly targeting Pin1. Biochim Biophys Acta 1843: 2055-2066, 2014.

26. Hong L, Han Y, Zhang H, Li M, Gong T, Sun L, Wu K, Zhao Q and Fan D: The prognostic and chemotherapeutic value of miR-296 in esophageal squamous cell carcinoma. Ann Surg 251: 1056-1063, 2010

27. Li T, Lu YY, Zhao XD, Guo HQ, Liu CH, Li H, Zhou L, Han YN, Wu KC, Nie YZ, et al: MicroRNA-296-5p increases proliferation in gastric cancer through repression of Caudal-related homeobox 1. Oncogene 33: 783-793, 2014.
28. Wei JJ, Wu X, Peng Y, Shi G, Basturk O, Yang X, Daniels G, Osman I, Ouyang J, Hernando E, et al: Regulation of HMGA1 expression by microRNA-296 affects prostate cancer growth and invasion. Clin Cancer Res 17: 1297-1305, 2011.

29. Manning BD and Cantley LC: AKT/PKB signaling: Navigating downstream. Cell 129: 1261-1274, 2007.

30. Agarwal E, Brattain MG and Chowdhury S: Cell survival and metastasis regulation by Akt signaling in colorectal cancer. Cell Signal 25: 1711-1719, 2013.

31. Kang B, Hao C, Wang H, Zhang J, Xing R, Shao J, Li W, $\mathrm{Xu} \mathrm{N}, \mathrm{Lu} \mathrm{Y}$ and Liu S: Evaluation of hepatic-metastasis risk of colorectal cancer upon the protein signature of PI3K/AKT pathway. J Proteome Res 7: 3507-3515, 2008.

32. Xu X, Sakon M, Nagano H, Hiraoka N, Yamamoto H, Hayashi N, Dono K, Nakamori S, Umeshita K, Ito Y, et al: Akt2 expression correlates with prognosis of human hepatocellular carcinoma. Oncol Rep 11: 25-32, 2004.

33. Wang L, Yao J, Sun H, Sun R, Chang S, Yang Y, Song T and Huang C: miR-302b suppresses cell invasion and metastasis by directly targeting AKT2 in human hepatocellular carcinoma cells. Tumour Biol 37: 847-855, 2016.

34. Liu LL, Lu SX, Li M, Li LZ, Fu J, Hu W, Yang YZ, Luo RZ, Zhang CZ and Yun JP: FoxD3-regulated microRNA-137 suppresses tumour growth and metastasis in human hepatocellular carcinoma by targeting AKT2. Oncotarget 5: 5113-5124, 2014. 